PROCEEDINGS OF THE

AMERICAN MATHEMATICAL SOCIETY

Volume 133, Number 6, Pages 1581-1586

S 0002-9939(04)07749-4

Article electronically published on December 6, 2004

\title{
HARMONIC ANALYSIS ON DISCRETE ABELIAN GROUPS
}

\author{
M. LACZKOVICH AND G. SZÉKELYHIDI \\ (Communicated by Andreas Seeger)
}

\begin{abstract}
Let $G$ be an Abelian group and let $\mathbb{C}^{G}$ denote the linear space of all complex-valued functions defined on $G$ equipped with the product topology. We prove that the following are equivalent.

(i) Every nonzero translation invariant closed subspace of $\mathbb{C}^{G}$ contains an exponential; that is, a nonzero multiplicative function.
\end{abstract}

(ii) The torsion free rank of $G$ is less than the continuum.

\section{INTRODUCTION}

Let $G$ be a locally compact Abelian group and let $C(G)$ denote the linear space of all complex-valued continuous functions defined on $G$ equipped with the topology of uniform convergence on compact sets. A nonzero function $m \in C(G)$ is called an exponential if $m$ is multiplicative; that is, if $m(x+y)=m(x) \cdot m(y)$ holds for every $x, y \in G$. It is easy to see that the translates of a function $f \in C(G)$ span a one-dimensional subspace of $C(G)$ if and only if $f$ is a constant multiple of an exponential. We say that harmonic (or spectral) analysis holds on $G$ if every nonzero translation invariant closed subspace of $C(G)$ contains an exponential. If the translation invariant closed subspaces of $C(G)$ are spanned by polynomialexponential functions, then we say that harmonic (or spectral) synthesis holds on $G$. (A function is polynomial-exponential if it belongs to the algebra generated by the continuous additive functions and by the exponentials.)

A theorem of R. J. Elliot [2] claims that harmonic synthesis holds on every discrete Abelian group. As it turned out, Elliot's proof was defective. In fact, by a recent result of L. Székelyhidi [6], the statement is false. Székelyhidi proves that harmonic synthesis fails on $\mathbb{R}$ (equipped with the discrete topology) or, more generally, on every Abelian group containing the free Abelian group of rank $\omega$. The statement that even harmonic analysis may fail on some free Abelian groups was proved in [5].

In this note our aim is to characterize those Abelian groups on which harmonic analysis holds. Let $r_{0}(G)$ denote the torsion free rank of $G$; that is, the cardinality of a maximal independent system of elements of infinite order. In other words, $r_{0}(G)$ is the maximal cardinality $\kappa$ such that $G$ contains the free Abelian group of

Received by the editors February 10, 2004.

2000 Mathematics Subject Classification. Primary 20K99; Secondary 43A45, 12 F05.

Key words and phrases. Problem of harmonic analysis, exponential functions, Hilbert's Nullstellensatz.

The research of the first author was partially supported by the Hungarian National Foundation for Scientific Research, Grant No. T032042. 
rank $\kappa$ as a subgroup. The cardinality of continuum is denoted by $2^{\omega}$. Our main result is the following.

Theorem 1. Harmonic analysis holds on a discrete Abelian group $G$ if and only if $r_{0}(G)<2^{\omega}$.

In particular, harmonic analysis holds on every discrete Abelian group of cardinality less than $2^{\omega}$ and fails to hold on every torsion-free Abelian group of cardinality $\geq 2^{\omega}$.

As a special case we find that harmonic analysis holds on the free Abelian group of rank $\omega$. (This was first proved in [4].) Since, by [6, harmonic synthesis fails to hold on this group, we find that harmonic analysis does not imply harmonic synthesis in general.

\section{SufFICIENCY}

In this section we show that if $r_{0}(G)<2^{\omega}$, then harmonic analysis holds on $G$. This will be shown by using duality.

The set of functions from $G$ to $\mathbb{C}$ will be denoted by $\mathbb{C}^{G}$. We shall say that an operator $D: \mathbb{C}^{G} \rightarrow \mathbb{C}^{G}$ is a difference operator if there are elements $a_{i} \in \mathbb{C}$ and $x_{i} \in G(i=1, \ldots, n)$ such that

$$
(D f)(x)=\sum_{i=1}^{n} a_{i} f\left(x+x_{i}\right)
$$

for every $f \in \mathbb{C}^{G}$ and $x \in G$. The set of all difference operators will be denoted by $\mathcal{D}$. If $A \in \mathcal{D}$ and $a \in \mathbb{C}$, then we define $a A \in \mathcal{D}$ by $(a A) f=a(A f)$. If $A, B \in \mathcal{D}$, then the sum and product of $A, B$ are defined by $(A+B) f=A f+B f$ and $(A B) f=A(B f)$. It is easy to check that under these operators $\mathcal{D}$ becomes a commutative algebra. Let $T_{g}$ denote the translation operator $T_{g} f(x)=f(x+g)$. Then $T_{0}$ is the identity operator; also, it is the unit element of $\mathcal{D}$. Every difference operator is the linear combination of translation operators.

The linear space $\mathbb{C}^{G}$ endowed with the product topology is a locally convex topological vector space. It is easy to see that the continuous linear functionals on $\mathbb{C}^{G}$ are of the form $L f=\sum_{i=1}^{n} a_{i} f\left(x_{i}\right)$, where $a_{i} \in \mathbb{C}$ and $x_{i} \in G(i=1, \ldots, n)$. Let $V$ be a translation invariant closed subspace of $\mathbb{C}^{G}$, and put $\mathcal{I}=\{D \in \mathcal{D}$ : $D f=0$ for every $f \in V\}$. Then $\mathcal{I}$ is an ideal of $\mathcal{D}$, and for every $f \in \mathbb{C}^{G}$ we have $f \in V \Longleftrightarrow D f=0(D \in \mathcal{I})$. Indeed, if $f \notin V$, then the local convexity of the space implies the existence of a continuous linear functional $L$ such that $L f \neq 0$ and $L g=0$ for $g \in V$. Thus there are elements $a_{i} \in \mathbb{C}$ and $x_{i} \in G(i=1, \ldots, n)$ satisfying $\sum_{i=1}^{n} a_{i} f\left(x_{i}\right) \neq 0$ and $\sum_{i=1}^{n} a_{i} g\left(x_{i}\right)=0$ for every $g \in V$. Since $V$ is translation invariant, we obtain $D=\sum_{i=1}^{n} a_{i} T_{x_{i}} \in \mathcal{I}$ and $D f \neq 0$. Clearly, if $V$ is nonzero, then $\mathcal{I} \neq \mathcal{D}$.

In order to prove that every nonzero translation invariant closed subspace contains an exponential, it is enough to show that if $\mathcal{I}$ is a proper ideal, then there is an exponential $m$ such that $D m=0$ for every $D \in \mathcal{D}$. The situation is that of Hilbert's Nullstellensatz, and the statement for the free group of rank $\omega$ can be proved by generalizing Hilbert's theorem to the polynomial ring of countably many variables [4]. In the general case we shall apply the proof of Hilbert's theorem instead of generalizing it. The next lemma is a variant of [3, Theorem 5.2, p. 32] 
and is contained in Problem 10(b) of [1, Chapter V. §3, p. 373]. For the sake of completeness we provide a simple proof.

Lemma 2. Suppose that $K$ is a field, $k$ is a subfield, and $X$ is a subset of $K$ such that $\max (|X|, \omega)<|k|$, and $K=k[X]$; that is, $K$ is generated as a ring by $k$ and $X$. Then $K$ is an algebraic extension of $k$.

Proof. It is enough to prove that every element of $X$ is algebraic over $k$. Suppose this is not true, and let $Y \neq \emptyset$ be a maximal algebraically independent subset of $X$. If $L=k(Y)$, then $k \subset L \subset K$, and every element of $K$ is algebraic over $L$. Let $p_{z}$ denote the monic minimum polynomial of $z \in K$ over $L$. Its coefficients are elements of $L$; that is, they are rational functions of the elements of $Y$ with coefficients from $k$. Let $G_{z} \in k[Y]$ be the product of the denominators of the coefficients of $p_{z}$. In the algebraic closure $\bar{L}$ of $L$ the polynomial $p_{z}$ splits into linear factors. The roots of these factors are the conjugates of $z$ over $L$. Clearly, the elementary symmetric polynomials of these conjugates are of the form $F / G_{z}$, where $F \in k[Y]$. Therefore, every symmetric polynomial of the conjugates of $z$ with coefficients from $k$ is of the form $F /\left(G_{z}\right)^{N}$, where $F \in k[Y]$ and $N$ is a positive integer.

Let $y_{0}$ be a fixed element of $Y$ (note that $Y \neq \emptyset$ by assumption). For every $z \in K$, the set $\Gamma_{z}$ of elements $c \in k$ such that the polynomial $G_{z}$ is divisible by $y_{0}-c$ is finite. Therefore, $\left|\bigcup_{x \in X} \Gamma_{x}\right| \leq \max (|X|, \omega)<|k|$, and thus there exists an element $c \in k$ such that $y_{0}-c$ is not a factor of any of the polynomials $G_{x}(x \in X)$.

Now $1 /\left(y_{0}-c\right) \in L \subset K=k[X]$, and thus $1 /\left(y_{0}-c\right)=p\left(x_{1}, \ldots, x_{n}\right)$, where $p$ is a polynomial of the elements $x_{1}, \ldots, x_{n} \in X$ with coefficients from $k$. Let $L_{1}=L\left(x_{1}\right) \ldots\left(x_{n}\right)$; then $L_{1}$ is a finite extension of $L$. Let $\phi_{j}(j=1, \ldots, N)$ be a list of the isomorphisms mapping $L_{1}$ into $\bar{L}$ and fixing the elements of $L$. Then

$$
N /\left(y_{0}-c\right)=\sum_{j=1}^{N} p\left(\phi_{j}\left(x_{1}\right) \ldots \phi_{j}\left(x_{n}\right)\right) \text {. }
$$

For each $i=1, \ldots, n$, the right-hand side is a symmetric polynomial of the conjugates of $x_{i}$ over $L$, and thus it is of the form $F / G$, where $F, G \in k[Y]$, and $G$ divides a power of $\prod_{i=1}^{n} G_{x_{i}}$. Since $L$ is a pure transcendental extension of $k$, it follows from $N /\left(y_{0}-c\right)=F / G$ that $G$ is divisible by $y_{0}-c$, and thus at least one of the polynomials $G_{x_{i}}$ must be divisible by $y_{0}-c$. This, however, contradicts the choice of $c$, which completes the proof.

Theorem 3. If $G$ is an Abelian group satisfying $r_{0}(G)<2^{\omega}$, then harmonic analysis holds in $G$.

Proof. As we saw already, it is enough to show that if $\mathcal{I}$ is a proper ideal, then there exists an exponential $m$ such that $D m=0$ for every $D \in \mathcal{I}$. We may assume that $\mathcal{I}$ is maximal. Then $K=\mathcal{D} / \mathcal{I}$ is a field. It is easy to see that $c \mapsto c \cdot T_{0}(c \in \mathbb{C})$ is an isomorphism from $\mathbb{C}$ into $\mathcal{D}$. Therefore, $k=\left\{c \cdot T_{0}: c \in \mathbb{C}\right\}$ is a subfield of $\mathcal{D}$ isomorphic to $\mathbb{C}$. Let $\phi$ denote the natural ring homomorphism from $\mathcal{D}$ onto $K$. Then $c \cdot T_{0} \mapsto \phi\left(c \cdot T_{0}\right)(c \in \mathbb{C})$ is an isomorphism from $k$ into $\mathrm{K}$, since $c \neq 0$ implies $c \cdot T_{0} \notin \mathcal{I}$ and $\phi\left(c \cdot T_{0}\right) \neq 0$. Thus $k^{\prime}=\phi(k)$ is a subfield of $K$ isomorphic to $\mathbb{C}$. If we identify $k^{\prime}$ with $\mathbb{C}$, then $K$ becomes an extension of $\mathbb{C}$.

We put $m(g)=\phi\left(T_{g}\right)$ for every $g \in G$. Then $m: G \rightarrow K$ is multiplicative since

$$
m(g+h)=\phi\left(T_{g+h}\right)=\phi\left(T_{g} T_{h}\right)=\phi\left(T_{g}\right) \cdot \phi\left(T_{h}\right)=m(g) m(h)
$$


for every $g, h \in G$. Also, $m(g) \neq 0$ for every $g$ since

$$
[m(g)=0] \Longrightarrow\left[T_{g} \in \mathcal{I}\right] \Longrightarrow\left[T_{0} \in \mathcal{I}\right] \Longrightarrow[\mathcal{I}=\mathcal{D}],
$$

which is not the case. If $D \in \mathcal{I}, D=\sum_{i=1}^{k} c_{i} T_{g_{i}}$, then we have

$$
\begin{aligned}
D m(g) & =\sum_{i=1}^{k} c_{i} \cdot m\left(g+g_{i}\right)=\sum_{i=1}^{k} c_{i} \cdot m(g) \cdot m\left(g_{i}\right)=m(g) \cdot \sum_{i=1}^{k} c_{i} \cdot \phi\left(T_{g_{i}}\right) \\
& =m(g) \cdot \phi(D)=0 .
\end{aligned}
$$

In order to complete the proof it is enough to show that $K=\mathbb{C}$. Clearly, $K=$ $\mathbb{C}[m(G)]$. In other words, $K$ is the ring generated by $\mathbb{C}$ and by the elements $m(g)(g \in G)$.

Let $t G$ denote the torsion group of $G$ (that is, the set of elements of finite order), and let $X \subset G$ be a set containing exactly one element of each coset of $t G$. We show that $|X|<2^{\omega}$. Suppose $|X| \geq 2^{\omega}$; then $|G / t G|=|X| \geq 2^{\omega}$. Now $G / t G$ is a torsion free group, and thus it contains a free Abelian group of rank $|G / t G| \geq 2^{\omega}$. Let $Y$ be a set of generators of this free subgroup with $|Y| \geq 2^{\omega}$. Let $\psi$ denote the natural homomorphism from $G$ onto $G / t G$, and select a point from each set $\psi^{-1}(\{y\})(y \in Y)$. The set $Z$ of these points is an independent system of elements of infinite order. Since $|Z| \geq 2^{\omega}$, it follows that $r_{0}(G) \geq 2^{\omega}$, contradicting the assumption on $G$. Thus $|X|<2^{\omega}$, as we stated.

Every element of $G$ can be written in the form $x+h$ where $x \in X$ and $h \in t G$. If $h \in t G$ and $n h=0$, then $1=m(0)=m(n h)=m(h)^{n}$ and thus $m(h)$ is an $n^{\text {th }}$ root of unity. In particular, $m(h) \in \mathbb{C}$ for every $h \in t G$. Therefore we have $K=\mathbb{C}[m(G)]=\mathbb{C}[m(X)]$. Since $|m(X)| \leq|X|<2^{\omega}=|\mathbb{C}|$, it follows from Lemma 2 that $K$ is an algebraic extension of $\mathbb{C}$. As $\mathbb{C}$ is algebraically closed, we obtain $K=\mathbb{C}$, which completes the proof.

\section{NECESSITY}

Lemma 4. If harmonic analysis holds on the group $G$, then the same is true for every subgroup of $G$.

Proof. Let $H$ be a subgroup of $G$, and let $V \neq 0$ be a closed invariant subspace of $\mathbb{C}^{H}$. Then $W=\left\{f \in \mathbb{C}^{G}:\left(T_{g} f\right) \mid H \in V\right.$ for every $\left.g \in G\right\}$ is a closed invariant subspace of $\mathbb{C}^{G}$. Let $U$ be a subset of $G$ containing exactly one element of each coset of $H$. If $f \in V, f \neq 0$, then the function $g: G \rightarrow \mathbb{C}$ defined by $g(u+x)=$ $f(x)(u \in U, x \in H)$ belongs to $W$, and thus $W \neq 0$. By assumption, $W$ contains an exponential $m$. Then $(m \mid H) \in V$, showing that harmonic analysis holds on $H$.

Lemma 5. Let $\mathcal{I}$ be a proper ideal of $\mathcal{D}$. Then there exists a function $f: G \rightarrow \mathbb{C}$ such that $f$ is not identically zero and $D f=0$ for every $D \in \mathcal{I}$.

Proof. Since $\mathcal{D}$ is a linear space over $\mathbb{C}, \mathcal{I}$ is a linear subspace of $\mathcal{D}$ and $T_{0} \notin \mathcal{I}$, there is a linear map $\psi: \mathcal{D} \rightarrow \mathbb{C}$ such that $\psi(D)=0$ for every $D \in \mathcal{I}$, and $\psi\left(T_{0}\right)=1$. We define

$$
f(g)=\psi\left(T_{g}\right)
$$

for every $g \in G$. Then $f$ maps $G$ into $\mathbb{C}$. Since $f(0)=\psi\left(T_{0}\right)=1, f$ is not identically zero. 
Let $D=\sum_{i=1}^{n} a_{i} T_{g_{i}}$ be an element of $\mathcal{I}$, and let $g \in G$. Then $\psi\left(T_{g} D\right)=0$ by $T_{g} D \in \mathcal{I}$, and thus

$$
\begin{aligned}
(D f)(g) & =\sum_{i=1}^{n} a_{i} f\left(g_{i}+g\right)=\sum_{i=1}^{n} a_{i} \cdot \psi\left(T_{g_{i}+g}\right) \\
& =\psi\left(\sum_{i=1}^{n} a_{i} T_{g} T_{g_{i}}\right) \\
& =\psi\left(T_{g} \cdot D\right)=0 .
\end{aligned}
$$

Thus $D f=0$ for every $D \in \mathcal{I}$, which proves the lemma.

If $r_{0}(G) \geq 2^{\omega}$, then $G$ contains the free Abelian group of rank $2^{\omega}$ as a subgroup. If harmonic analysis held on $G$ then, by Lemma 4, it would hold on each subgroup as well. Thus the next lemma will complete the proof of Theorem 1. Note that the statement of the lemma is just a reformulation of Problem 10(c) of [1, Chapter V. $\S 3$, p. 373]. Again, we provide the simple proof.

Lemma 6. Let $G$ be a free Abelian group of rank $\geq 2^{\omega}$. Then harmonic analysis does not hold on $G$.

Proof. Let $X$ be the set of generators of $G$. Since $|X|=|G| \geq 2^{\omega}$, there exists a surjective map $\phi: X \rightarrow \mathbb{C}(t)$. Then there is a unique homomorphism $\Phi: \mathcal{D} \rightarrow \mathbb{C}(t)$ such that $\Phi\left(T_{x}\right)=\phi(x)$ for every $x \in X$. Put

$$
\mathcal{J}=\{D \in \mathcal{D}: \Phi(D)=0\} .
$$

Since $\mathbb{C}(t)$ is a field, $\mathcal{J}$ is a maximal ideal, and the factor ring $\mathcal{D} / \mathcal{J}$ is isomorphic to $\mathbb{C}(t)$. The set of functions

$$
V=\left\{f \in \mathbb{C}^{G}: D f=0(D \in \mathcal{J})\right\}
$$

is a closed translation invariant subspace of $\mathbb{C}^{G}$ and, by Lemma 5$] V$ is nonzero. We claim that $V$ does not contain any exponential function. Suppose $m \in V$ is an exponential. It is easy to check that the map

$$
\Psi\left(\sum_{i=1}^{n} a_{i} T_{b_{i}}\right)=\sum_{i=1}^{n} a_{i} m\left(b_{i}\right)
$$

is a homomorphism from $\mathcal{D}$ into $\mathbb{C}$, and $D m=0$ if and only if $\Psi(D)=0$. The ideal $\mathcal{I}=\{D \in \mathcal{D}: D m=0\}$ contains $\mathcal{J}$ and hence $\mathcal{I}=\mathcal{J}$ by the maximality of $\mathcal{J}$. Thus $\mathcal{J}$ is the kernel of $\Psi$. Therefore, $\mathcal{D} / \mathcal{J}$ is isomorphic to $\mathbb{C}$, which is a contradiction.

\section{REFERENCES}

[1] N. Bourbaki: Commutative Algebra. Hermann and Addison-Wesley, 1972. MR0360549 (50:12997)

[2] R. J. Elliot, Two notes on spectral synthesis for discrete Abelian groups, Proc. Cambridge Phil. Soc. 61 (1965), 617-620. MR0177260 (31:1523)

[3] H. Matsumura: Commutative Ring Theory. Cambridge University Press, 1986. MR0879273 (88h:13001)

[4] G. Székelyhidi, Spectral synthesis on locally compact Abelian groups (essay). Cambridge, Trinity College, 2001. 
[5] G. Székelyhidi, Spectral analysis, unpublished manuscript, 2002.

[6] L. Székelyhidi, The failure of spectral synthesis on some types of discrete Abelian groups, J. Math. Anal. and Applications 291 (2004), no. 2, 757-763. MR2039084

Department of Analysis, Eötvös Loránd University, Budapest, Pázmány Péter sétány 1/C, 1117 Hungary - and - Department of Mathematics, University College London, Gower Street, London, WC1E 6BT, England

E-mail address: laczk@cs.elte.hu

Department of Mathematics, Imperial College, Huxley Building, 180 Queen's Gate, LONDON, SW7 2AZ, ENGLAND

E-mail address: gabor.szekelyhidi@imperial.ac.uk 